\title{
Experimental and DEM analysis of the dissipation involved in the collision of a boulder with a substratum
}

\author{
Bruna Garcia ${ }^{1,2, \star}$, Pascal Villard ${ }^{1}$, Vincent Richefeu ${ }^{1}$, and Dominique Daudon ${ }^{1}$ \\ ${ }^{1}$ Univ. Grenoble Alpes, 3SR, F-38000, Grenoble, France \\ ${ }^{2}$ IMSRN, Ingénierie des Mouvements de Sol et Risques Naturels, 680 Rue Aristides Bergès, 38330 Montbonnot, France
}

\begin{abstract}
Experiments and numerical analysis were both carried out to evaluate the amount of energy dissipated during the rebound between single boulder (concrete made blocks with many varied shapes) and a soft or stiff substratum. A high speed camera made it possible to analyze the collision kinematics and to determine the role played by different features such as the incident axial/angular velocities, the boulder shapes, the impact configuration and the substratum type, on the amount of dissipated energy. A three-dimensional discrete element code was used to simulate the experiments by accounting for the actual shape of the boulder and some dissipation mechanisms. The comparison between the experimental and numerical trajectories allowed for an physical interpretation of the dissipation mechanisms and an identification of a set of parameters to be introduced in the simulations. That way, the predictive ability of the discrete model of rock avalanche can be improved by including in-situ measurements.
\end{abstract}

\section{Introduction}

Rock falls or rock avalanches are becoming increasingly frequent due to climatic changes. Economic issues resulting from the closure of roads or the discontinuation of economic activities can be significant and their consequences on people and constructions are sometimes dramatic. The prediction of the propagation way and the stop position of a rock is highly uncertain given the complexity of the involved mechanisms. For a better understanding of such mechanisms, a numerical model was developed to take into account the quantities and modes of dissipated energies during the rebound of a rock on a smooth or a rigid substratum. The numerical model used is based on the DEM approach and takes account of real shape of blocks and energy dissipation modes due to collisions, friction and rolling resistance $[1,4]$. First validated with experimental data resulting from small scale laboratory experiments involving the rebound of small parallelepiped blocks on a rigid substratum [2,3], the ability of the numerical model to predict the rebound of a rock on a natural soil was evaluated using large scale laboratory experiments involving different block shapes. After validation, the numerical model could be used to conduct a statistic analysis of the influence of the block shape that was not possible in an experimental way due to the large number of tests that were needed. In this paper we focus on the methodology used to obtain the optimized set of parameters from experimental tests.

\section{Experimental tests}

The device shown in Figure 1 is basically a metallic ramp in which a released boulder (1) rolls or slides, (2) flies freely, (3) collides with a substratum, and (4) bounces. The metal ramp is 2.7 meters long and its inclination can vary from 30 to 60 degrees, reaching a maximum elevation height of $2.30 \mathrm{~m}$.

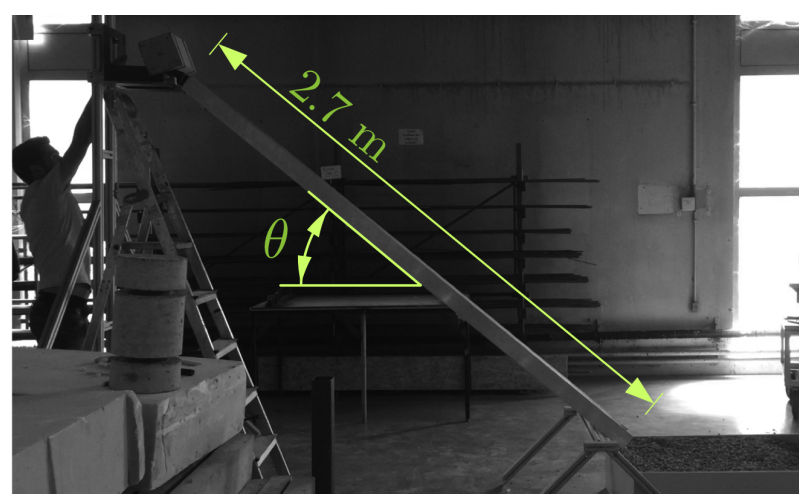

Figure 1. Experimental device. The inclination angle $\theta$ can vary from $30^{\circ}$ to $60^{\circ}$.

The boulder kinematics is restricted to a vertical plan so that the stages 2 to 4 can be filmed with a single highspeed camera. Photographs were shot each 0.1 second to determine the boulder trajectories before and after impact. Both axial and angular positions were then tracked on the photographs thanks to two reference points marked in the visible face of the boulder.

\footnotetext{
^e-mail: bruna.dasilvagarcia@3sr-grenoble.fr
} 
All the lengths were scaled by using the pixel sizes, which depends on the position and settings of the camera. The framework origin was arbitrarily set at the first collision point on the substratum plan; Figure 2. As a convenient consequence, $(i)$ the incident trajectory has negative $x$-coordinates while the reflected trajectory has a positive $x$-coordinates; (ii) a positive $y$-coordinate corresponds to free flight motions, and a negative $y$-coordinate plays the same role as an overlap in discrete element simulations, and should be associated to the "contact phase".

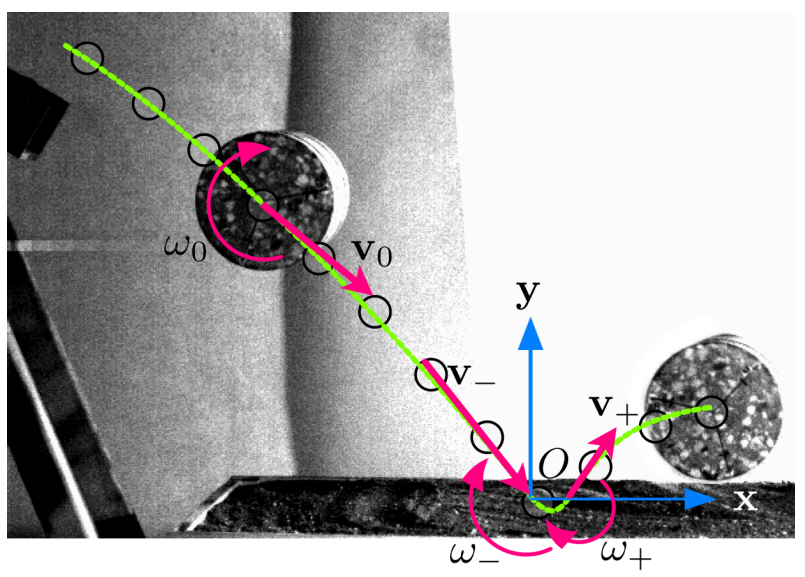

Figure 2. Sketch of the assessment of the trajectories before and after a collision. The circle symbols are the positions extracted from the picture shots; the green curve is the trajectory that can be obtained by means of statistical regression; $\mathbf{V}_{0}$ and $\omega_{0}$ are initial velocities that can be used to perform a DEM simulation; $\mathbf{V}_{-}$and $\omega_{-}$are the incident velocities, and $\mathbf{V}_{+}$and $\omega_{+}$are the reflected velocities.

Many geometries have been designed (Figure 3); the boulders have the same height $(0.25 \mathrm{~m})$ and they are made of ultra-high performance fiber-reinforced concrete (UHPC) in order to avoid their breakage. Luckily, the density of UHPC $\left(\sim 2400 \mathrm{~kg} / \mathrm{m}^{3}\right)$ is close to the average density of natural rocks $\left(\sim 2700 \mathrm{~kg} / \mathrm{m}^{3}\right)$. Two grooves intended to guide the boulder on the rail were designed so that the rotation was locked or free. The boulder weight $m$, axial inertia $J$ and rotation hindrance in the ramp are listed in Table 1.

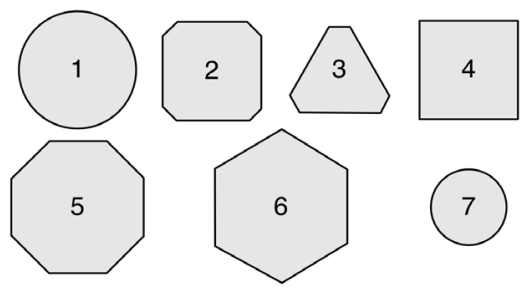

Figure 3. Boulder shapes used for the tests: (1) cylinder, $16 \mathrm{~cm}$ in diameter; (2) square with trimmed-corners, $150 \mathrm{~mm}$ in sides; (3) triangle with trimmed-corners, $155 \mathrm{~mm}$ in sides; (4) square, $160 \mathrm{~mm}$ in sides; (5) octagon, $400 \mathrm{~mm}$ in outer-diameter; (6) hexagon, $185 \mathrm{~mm}$ in outer-diameter; (7) cylinder, $110 \mathrm{~mm}$ in diameter.
Table 1. Mass properties of the boulders and ability to roll when released on the ramp.

\begin{tabular}{crcc}
\hline $\begin{array}{c}\text { Shape } \\
\text { number }\end{array}$ & $\begin{array}{r}\text { Weight } \\
m(\mathrm{~kg})\end{array}$ & $\begin{array}{c}\text { Axial inertia } \\
J\left(\mathrm{~kg} \cdot \mathrm{m} \cdot \mathrm{s}^{2}\right)\end{array}$ & $\begin{array}{c}\text { Rotation } \\
\text { locked }\end{array}$ \\
\hline 1 & 10.89 & 0.021820 & No \\
2 & 11.82 & 0.040750 & No \\
3 & 11.66 & 0.038314 & No \\
4 & 13.80 & 0.060325 & Yes \\
5 & 33.52 & 0.345403 & No \\
6 & 16.92 & 0.087076 & No \\
7 & 5.38 & 0.008177 & No \\
\hline
\end{tabular}

To quantify the global dissipation as a function of the boulder shape and of the substratum, the restitution rate of the total kinetic energy has been determined from the axial velocities $\mathbf{v}$ and angular velocities $\omega$, before (-) and after (+) the collision (Figure 2):

$$
R=\frac{m \mathbf{v}_{+}^{2}+J \omega_{+}^{2}}{m \mathbf{v}_{-}^{2}+J \omega_{-}^{2}}
$$

In practice, the time "just before" or "just after" the rebound is a matter of choice because the collision has a finite duration, which cannot be uniquely defined from the photographs. We however checked that a small variation in the delays before and after the rebound for the assessment of velocities has a very limited influence on the value of the restitution rate $R$. Thus, the last/first known velocity belonging to a parabolic trajectory of the mass center (free flight) were used for the $-/+$ values (respectively) in Equation 1.

Table 2 presents the restitution rates $R$ for the 7 different shapes. All releases were performed from the same high and with a ramp inclination $\theta=45^{\circ}$. Except for shape 4 that slid on the ramp, the other shapes rolled. The impacted horizontal substratum was either a dry and loose sand (LS), a stabilized soil (SS), a bituminous concrete (BC) or a vegetated ground (VG).

From the data in Table 2, a number of facts can be drawn. The reproducibility for each drop is not so good excepted for the collision with the loose sand (LS), which is the softest substratum. This lack of repeatability may be due to geometrical or mechanical heterogeneities, however, the same order of $R$ was obtained - at least two times over the 3 drops - for nearly all couples of shapesubstratum. It is clear that both the shape of the boulder and the nature of the substratum affect the amount of kinetic energy restored after the collision. However, we stress here that the restitution rate $R$ makes no distinction on how the kinetic energy is dissipated. For example, it does not evaluate what is the part of incident kinetic energy that has been transformed in angular velocity. As a consequence, an identification of the dissipation parameters involved in a model of collision may help to understand the mechanisms that take place as a function of the boulder shape and of the substratum. 
Table 2. Values of the restitution rate $R$ (see Equation 1) for 3 drops of different shapes on a ramp inclined at $45^{\circ}$. Four types of substratum were tested: Loose and dry Sand (LS), Bituminous Concrete (BC), Vegetated Ground (VG) and Stabilized Soil (SS).

\begin{tabular}{ccccc}
\hline Shape & Substratum & Drop 1 & Drop 2 & Drop 3 \\
\hline \multirow{4}{*}{1} & LS & 0.45 & 0.36 & 0.49 \\
& SS & 0.50 & 0.74 & 0.58 \\
& BC & 0.62 & 0.94 & 0.65 \\
& VG & 0.58 & 0.35 & 0.95 \\
\hline \multirow{2}{*}{2} & LS & 0.48 & 0.58 & 0.67 \\
& BC & 0.40 & 0.76 & 0.46 \\
\hline \multirow{3}{*}{3} & LS & 0.39 & 0.56 & - \\
& SS & 0.58 & 0.26 & - \\
& VG & 0.34 & 0.10 & 0.34 \\
\hline \multirow{4}{*}{4} & LS & 0.62 & 0.63 & 0.62 \\
& SS & 0.41 & 0.35 & 0.45 \\
& BC & 0.49 & 0.49 & 0.39 \\
& VG & 0.38 & 0.23 & 0.29 \\
\hline \multirow{2}{*}{5} & LS & 0.41 & 0.45 & $($ broken) \\
& BC & 0.88 & 0.54 & 0.45 \\
& VG & 0.68 & 0.82 & - \\
\hline \multirow{2}{*}{6} & LS & 0.28 & 0.28 & - \\
& BC & 0.50 & 0.47 & 0.52 \\
& VG & 0.82 & 0.73 & 0.64 \\
\hline 7 & BC & 0.65 & 0.41 & 0.47 \\
\hline
\end{tabular}

\section{Collision parameters}

A boulder trajectory can be computed by means of the discrete element method (DEM) and then compared with the $2 \mathrm{D}$ experimental trajectory. When the initial conditions (axial/angular velocities and positions) are correctly set, the computed trajectory matches the experimental one within a given tolerance. When the boulder leaves the horizontal plan that represents the substratum surface, the computed trajectory relies on the collision parameters. Here, the objective is to determine the DEM parameters that imply the reflected velocities having the closer match with the measured ones.

These DEM parameters are those of a local dissipative contact model developed for rock avalanches (see e.g. [1] or [2]), implemented in the code DEMbox. They are (1) the normal, tangential and rolling stiffnesses $k_{n}, k_{t}$ and $k_{r}$, respectively, and (2) the associated dissipation coefficients, namely:

- The kinetic energy restitution $e_{n}^{2}$ in the normal direction.

- The tangential dissipation coefficient $\mu$ that relates to the friction and/or to the plasticity of the soil. Notice that $\mu$ is not named "friction coefficient" and it may be greater than 1 .

- The rolling dissipation coefficient $\mu_{r}$ that acts like $\mu$ but for the rolling movements. Notice that this latter parameter is dimensionless.

Since the simulation results should only differ from the experiments after the collision, the relative-error functions are based on the reflected velocities, that are the axial and angular velocities of the boulder when it leaves the horizontal plan. For each component $x$ of reflected velocity ( $x$ stands for either $v_{x}, v_{y}, \omega$ or $R$ ), it expresses as follows:

$$
E_{x}(\{p\})=\left(\frac{x_{+}^{\mathrm{sim}}-x_{+}^{\exp }}{x_{+}^{\exp }}\right)^{2}
$$

where $\{p\}=\left(k_{n}, k_{t}, k_{r}, e_{n}^{2}, \mu\right.$ and $\left.\mu_{r}\right)$ is the set of collision parameters.

\section{Selected results}

Finding the best parameters $\{p\}$ can be done by optimizing them with a multivariate minimization technique like, e.g., the conjugate directions descent or Powell's method. We, however, chose to use a brute-force optimization because it can inform us about what are the effects of varying each parameter independently. Two examples of such analyses are presented here for two different materials of the substratum: dry-loose sand (LS) and stabilized soil (SS). Both examples make use of the shape 1, that is a cylinder with $16 \mathrm{~cm}$ in diameter. The incident and reflected velocities, measured from the photographs of the experiments, are given in Table 3 .

Table 3. Incident and reflected velocities extracted from the photographs, for the two cases selected. The values into brackets are suspicious since the horizontal velocity should not be increased with cylindrical boulders; in this case it was chosen in the optimization procedure $v_{x+}=v_{x-}=(3.17+3.32) / 2$.

\begin{tabular}{ccccccc}
\hline & $v_{x-}$ & $v_{y-}$ & $\omega_{-}$ & $v_{x+}$ & $v_{y+}$ & $\omega_{+}$ \\
& $\mathrm{m} / \mathrm{s}$ & $\mathrm{m} / \mathrm{s}$ & $\mathrm{s}^{-1}$ & $\mathrm{~m} / \mathrm{s}$ & $\mathrm{m} / \mathrm{s}$ & $\mathrm{s}^{-1}$ \\
\hline Soft & 3.69 & -3.29 & -50.52 & 3.22 & 0.49 & -37.09 \\
Stiff & $(3.17)$ & -3.51 & -37.74 & $(3.32)$ & 0.44 & -40.80 \\
\hline
\end{tabular}

For the brute-force optimization of the collision parameters between the cylinder and the loose sand (LS), a number of preliminary simulations have been carried out. The resulting trajectories have been compared with the experimental one, and it was found that mainly 3 parameters had a major influence on the vertical, horizontal and angular velocities; they are respectively $e_{n}^{2}, \mu$ and $\mu_{r}$. The tangential and angular stiffnesses have been set to high values so that the corresponding efforts are rapidly mobilized. The normal contact stiffness $k_{n}$ was adapted to the type of the substratum in order to reproduce similar ground settlements than those obtained experimentally $\left(k_{n} \simeq 10^{6} \mathrm{kN} / \mathrm{m}\right.$ and $k_{n} \simeq 10^{7} \mathrm{kN} / \mathrm{m}$ for the soft and stiff soils, respectively).

Noticing that $e_{n}^{2}$ is the only parameter that affects the vertical velocity of the boulder, it was chosen to identify first this parameter. Figure 4 shows the relative-error function $E_{v_{y}}$ as a function of $e_{n}^{2}$. We observe that the reflected vertical velocity is unambiguously optimized for $e_{n}^{2} \simeq 0.04$ with a zero error (whatever the values of $\mu$ and $\mu_{r}$ ).

The influence of $\mu$ and $\mu_{r}$ on the horizontal and angular velocities can be examined in a two-dimensional space as shown in Figure 5, where $e_{n}^{2}$ has been set to its optimum value. The horizontal velocity is shown to be controlled 


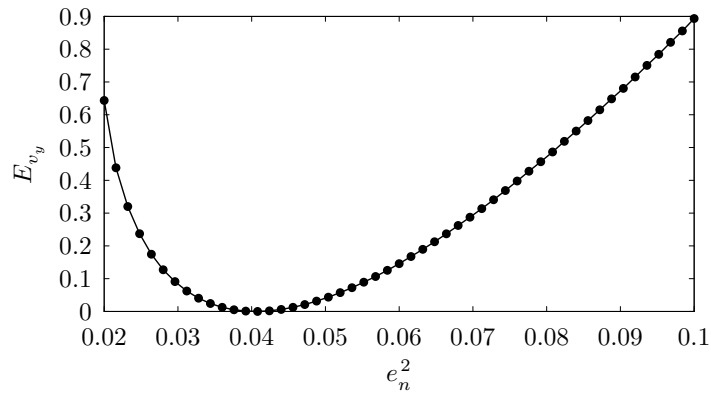

Figure 4. Relative-error function $E_{v_{y}}$ as a function of $e_{n}^{2}$ in the case of a collision with a soft substratum.

only by $\mu$ but unaffected by $\mu_{r}$. This is indeed expected since the horizontal component of the resultant force is the friction force, which dissipates by the only means of $\mu$ since the normal force is independently monitored. In contrast, the angular velocity seems affected by both $\mu$ and $\mu_{r}$ for the reason that the resultant moment of the cylindrical boulder is thresholded by the sum of two terms: (1) the resistant moment caused by friction $\ell\left(-\mu f_{n}\right)$, where $\ell$ is the lever arm (radius of the cylinder) and $f_{n}$ the vertical force of contact, and (2) the moment caused by rolling resistance $\mu_{r} \ell f_{n}$. It results that the resistant moment, and thus the changes in angular acceleration, is controlled by the value of $\left(\mu_{r}-\mu\right)$. This observation is reflected in Figure 5 by the zero-level isocurve of $E_{\omega}$ which is a straight line given by $\mu_{r}-\mu=0.08$.

Finally, for the studied collision of the cylindrical boulder on the loose sand, the optimum parameters are obtained at the intersection between the zero-level isocurves of $E_{v_{x}}$ and $E_{\omega}$, where $\mu \simeq 0.115, \mu_{r} \simeq 0.195$, and $e_{n}^{2} \simeq 0.04$.

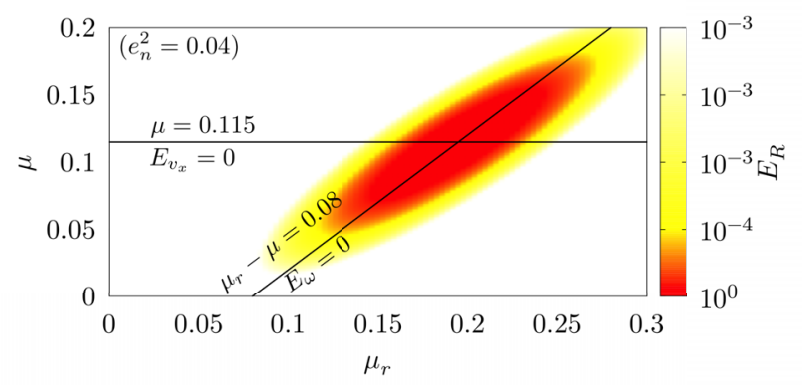

Figure 5. Zero-level isocurves of $E_{v_{x}}$ and $E_{\omega}$ in the space $\left(\mu-\mu_{r}\right)$, $e_{n}^{2}$ being set to 0.04 . These two curves are respectively defined by the relations $\mu=0.115$ and $\mu_{r}-\mu=0.08$; they intersect thus at $\mu_{r}=0.195$. The color map represents the relative error $E_{R}$ on the restitution rate $R$.

For the collision of the same boulder on a stabilized soil, a similar optimization procedure led to the following set of dissipation parameters: $\mu \simeq 0.05, \mu_{r} \simeq 0.0$ and $e_{n}^{2} \simeq$
0.04. In this case that involves a stiffer substratum, very low values of $\mu$ and $\mu_{r}$ were found because the horizontal and angular velocities were nearly unaffected. In fact, we noticed that an increase in the horizontal velocity was not obtainable because a very small value of $\mu$ caused only a slight increase in the angular velocity, but it also induced a sensible decrease in the horizontal velocity.

\section{Concluding remarks}

In this work, we intended to detect which of the 6 parameters implied in the proposed collision model were the most influential and caused the greater variations in reflected velocities after a collision. These parameters were found to be different depending on the nature of the substratum (soft or stiff), and some conclusions are now beginning to emerge. For example, the rolling resistance was found to be inoperative in case of stiff substratum, while it is absolutely required with soft substratum. Another noticeable observation is the very low restitution of energy in the normal direction whatever the stiffness of studied substratum. The boulder shape plays also a crucial role in the dissipation mechanisms but it was not investigated in the present short paper. For the two types of soils taken into account for the numerical analysis, it was found that most of the vertical kinetic energy was dissipated during the rebound while the horizontal and rotational kinematics energy were only affected in the case of the soft soil.

The study is not yet completed and further analysis are still in progress or scheduled. In particular, more couples shape-substratum will be analyzed, and each analysis will be repeated to assess the variability of the identified parameters.

The authors thank T. Verchier, C. Grardel, T. Boudry and A. Colson who assisted the realization of the experimental tests in the framework of their final year study project. They also thank G. Vian for the conception and realization of the ramp. This study is funded by the company IMSRN (www.imsrn.com). The Laboratoire 3SR is part of the LabEx Tec 21 (Investissements d'Avenir, Grant Agreement No. ANR-11-LABX-0030).

\section{References}

[1] V. Richefeu, G. Mollon, D. Daudon, P. Villard, Engineering Geology 149, pp. 78-92 (2012)

[2] V. Richefeu, P. Villard, Modeling Gravity Hazards from Rockfalls to Landslides (ISTE Press Ltd, Elvesier Ltd, UK, 2016) $161 \mathrm{pp.}$

[3] G. Mollon, V. Richefeu, P. Villard, D. Daudon, Journal of Geophysical Research - Earth Surface 117, F02036 (2012)

[4] D. Daudon, P. Villard, V. Richefeu, G. Mollon, C. R. Mécanique, Mechanics of granular and polycrystalline solids 343(2), pp. 166-177 (2015) 